\title{
Relationships between Physical and Cognitive Functioning and Activities of Daily Living in Children with Cerebral Palsy
}

\author{
Chiang-Soon Song, PhD, OT ${ }^{1)}$ \\ 1) Department of Occupational Therapy, Chungnam Provincial Cheongyang College: 55 Haksa-gil, \\ Cheongyang-eup, Cheongyang-gun, Chungcheongnam-do 345-702, Republic of Korea. \\ TEL: +82 41-940-6776, FAX: +82 41-940-6734
}

\begin{abstract}
Purpose] The purpose of the present study was to evaluate the relationship between physical function, cognitive function, and activities of daily living in children with cerebral palsy. [Subjects] Sixty-eight children who had been diagnosed with cerebral palsy and have participated in inpatient or outpatient rehabilitation programs were enrolled in the present study. We used 3 clinical assessments: the Gross Motor Function Measure (GMFM), the Bayley Infant Development Screening Test-II (BSID-II), and the Wee Functional Independence Measure (WeeFIM). [Results] The GMFM was positively correlated with the BSID-II motor scale and the BSID-II cognitive scale, but not with the WeeFIM scores. The BSID-II motor scale was significantly correlated with the GMFM and BSID-II cognitive scale, but not with the WeeFIM. [Conclusion] The results of this study provide evidence of the necessity of including cognitive and physical impairments in the examination and evaluation of children with cerebral palsy in research and clinical settings.

Key words: Activities of daily living, Cerebral palsy, Cognition
\end{abstract}

(This article was submitted Dec. 13, 2012, and was accepted Jan. 11, 2013)

\section{INTRODUCTION}

Cerebral palsy (CP) is a non-progressive disease of the central nervous system caused by immature brain damage before, during, or after birth; it results in both motor and cognitive impairment ${ }^{1}$. The prevalence of CP in USA was reported 2.7 per 1,000 persons in 2007 , and it has a gradually increasing trend. The estimated prevalence of $\mathrm{CP}$ among children has been increasing because the survival rate of high-risk, low-birth-weight children is greater than it was in the past due to the advent of modern medicine. The causes of $\mathrm{CP}$ greatly vary, but the main factors associated with $\mathrm{CP}$ are low birth weight, premature birth among low-birth-weight infants, neonatal asphyxia, and kernicterus. Impairments caused by CP include visual impairment, hearing impairment, epilepsy, motor weakness, mental retardation, emotional disturbance, distinctive types of learning disabilities, and brain injury ${ }^{2)}$.

Child development proceeds in a certain direction, and individual differences in differentiation and integration experiences affect each person throughout his or her life. Therefore, a multidimensional treatment approach might be appropriate for treating the developmental processes of children with $\mathrm{CP}^{3}$. To provide evidence and fundamental knowledge regarding treatment approaches, clinicians should understand both the physical and cognitive disabilities caused by $\mathrm{CP}$, and should know how such disabilities affect activities of daily living (ADL). Clinical tools that

E-mail: grsong@daum.net measure physical and cognitive functions include the Gross Motor Function Measure (GMFM), the Bayley Infant Development Screening test-II (BSID-II), and the Wee Functional Independence Measure (WeeFIM) $)^{4}$. These tools have been individually validated as reliable and valid assessment tools for CP. However, there have been few studies of the correlations between each function and $\mathrm{CP}$ status in children with CP. In order to prescribe a multifactorial, integrated therapeutic approach for children with $\mathrm{CP}$, and to predict the effects of physical and cognitive functions on ADL, therapists need to gather sufficient information and evidence about existing or potential problems with motor and cognitive functions, which are ultimately integrated with ADL in children with CP. However, the way in which cognition interacts with the rehabilitation process is controversial, and opinions on this topic are largely uninformed by empirical evidence related to children with $\mathrm{CP}^{5,6)}$.

The purpose of this study was to evaluate the relationship between physical function, cognitive function, and ADL in children with CP. This study also examined the effects of age, CP type, and the ability to stand with or without assistance on physical function, cognitive function, and ADL of children with CP. This study measured the GMFM, BSID-II, and WeeFIM to achieve these purposes.

\section{SUBJECTS AND METHODS}

Sixty-eight children who had been diagnosed with $\mathrm{CP}$ and had participated in inpatient or outpatient rehabilitation programs were enrolled in this study. Before the study 
Table 1. General characteristics of the subjects in this study $(\mathrm{N}=68)$

\begin{tabular}{lccc}
\hline Characteristics & Classification & Boys (\%) & Girls (\%) \\
\hline \multirow{4}{*}{ Age (month) } & $1-12$ & $5(7.4)$ & $1(1.5)$ \\
& $13-24$ & $10(14.7)$ & $11(16.2)$ \\
& $25-36$ & $8(11.8)$ & $9(13.2)$ \\
\multirow{4}{*}{ Types } & $37-42$ & $8(11.8)$ & $3(4.4)$ \\
& $\geq 43$ & $6(8.8)$ & $7(10.3)$ \\
& Spastic hemiplegia & $7(10.3)$ & $7(10.3)$ \\
Standing & Spastic diplegia & $13(19.1)$ & $7(10.3)$ \\
& Spastic quadriplegia & $12(17.6)$ & $13(19.1)$ \\
& Dystonia & $5(7.4)$ & $4(5.9)$ \\
& Independently & $19(27.9)$ & $10(14.7)$ \\
& With assistance & $18(26.5)$ & $21(30.9)$ \\
\hline
\end{tabular}

began, the parents of all the children were provided with information about the research purpose and protocol, and gave their voluntarily agreement to their children's participation in this study. The inclusion criteria for the study subjects were as follows: diagnosis of $\mathrm{CP}$ by a pediatrician, no other neurological disorders or musculoskeletal diseases, no serious medical complications, and without any inspections. The exclusion criteria were: unstable seizures, treatment for spasticity which had lasted for more than 3 months, severe cognitive impairment, taking antipsychotic preparations, or having undergone surgery within the last 6 months. The clinical characteristics of the participating children are described in Table 1.

All of the participants were measured using three clinical tools to assess their physical and cognitive functioning. The clinical tools used were the GMFM, BSID-II, and WeeFIM. The GMFM was developed by Russell, and is an assessment tool which measures the gross motor developmental status of children with CP. The items of the GMFM are appropriate for children whose motor skills are at or below those of a normal 5 year-old. The tool consists of 88 items rated on a 4-point ordinal scale: 0 points (completely failed to perform) to 3 points ( $100 \%$ done). The inter-rater reliability of this measure ranges from 0.92 to 0.99 for children with $\mathrm{CP}^{7}$ ). The BSID was modified from the California Infants' Developmental Test in order to evaluate the development of infants and toddlers according to features developed by Bayley. The tool consists of three subscales: cognitive (mental), movements (motor), and behavior scales. The test-retest reliability of this measure is 0.88 ; thus, it shows high reliability ${ }^{8}$. The WeeFIM is an 18-item observational instrument modeled on the adult Functional Independence Measure. It measures the severity of disability and changes in the functional abilities of children over time and in rehabilitation settings. The tool measures direct observations obtained through interviews or by the guardian of a child regarding exercise and the performance of cognitive tasks. The inter-rater reliability of this measure ranges from 0.82 to $0.94^{9)}$.

All assessments in this study were conducted in a quiet, uncluttered therapy room. Three therapists with more than 10 years' experience of evaluating children with $\mathrm{CP}$, performed the tests. The therapists evaluated the GMFM and BSID-II while watching video tapes, and the WeeFIM through interviews and observation of the child's performance. In addition, all measurements were completed within 10 days in order to exclude the impact of child development.

Physical function, ADL, cognitive function, and gross motor functions were compared in this study using the GMFM, BSID-II, and WeeFIM. The general characteristics of the participants were analyzed using descriptive statistics. Pearson's correlation coefficients were calculated to examine the effects of physical function on cognitive function and ADL in children with CP. The collected data were analyzed using SPSS version 17.0 for Windows (SPSS. Inc., Chicago, IL, USA), and significance was accepted for values of $\mathrm{p}<0.05$.

\section{RESULTS}

This study evaluated the effects of age, types of $\mathrm{CP}$, and the ability to stand with or without assistance on physical and cognitive functions and ADL in children with CP. Participants' scores on the GMFM, BSID-II motor scale, BSID-II cognitive scale, and WeeFIM did not significantly differ with age (Table 2). WeeFIM scores differed significantly with CP type. However, the GMFM and the motor and cognitive scales of BSID-II did not significantly differ with type of CP (Table 3). The GMFM, BSID-II motor scale, and BSID-II cognitive scale significantly differed between the ability to stand with and without assistance. However, WeeFIM scores did not significantly differ between the ability to stand with and without assistance (Table 4).

The GMFM was significantly, positively correlated with the BSID-II motor scale ( $\mathrm{r}=0.922)$ and the BSID-II cognitive scale $(r=0.719)$, but not with WeeFIM scores. The BSID-II motor scale was significantly, positively correlated with the GMFM ( $\mathrm{r}=0.719)$ and BSID-II cognitive scale $(\mathrm{r}=$ 0.866), but not with the WeeFIM (Table 5).

\section{DISCUSSION}

The present study examined the effects of age, type of CP, 
Table 2. Effects of age on the GMFM, BSID-II, and WeeFIM ( $\mathrm{N}=68)$

\begin{tabular}{ccccc}
\hline Age (month) & GMFM & $\begin{array}{c}\text { BSID-II } \\
\text { Motor scale }\end{array}$ & $\begin{array}{c}\text { BSID-II } \\
\text { Cognitive scale }\end{array}$ & WeeFIM \\
\hline $1-12$ & $45.8 \pm 28.3$ & $50.0 \pm 29.0$ & $89.0 \pm 53.7$ & $57.0 \pm 14.8$ \\
$13-24$ & $44.8 \pm 28.9$ & $51.1 \pm 24.6$ & $90.9 \pm 40.0$ & $64.3 \pm 29.4$ \\
$25-36$ & $42.2 \pm 26.6$ & $46.9 \pm 22.6$ & $86.4 \pm 38.9$ & $74.1 \pm 27.4$ \\
$37-42$ & $60.5 \pm 30.4$ & $68.1 \pm 27.1$ & $112.2 \pm 37.4$ & $84.7 \pm 24.7$ \\
$\geq 43$ & $42.0 \pm 28.1$ & $53.9 \pm 23.9$ & $100.9 \pm 42.2$ & $57.2 \pm 22.7$ \\
\hline
\end{tabular}

GMFM, Gross Motor Function Measure; BSID-II, Bayley Scale Infant Development-II; WeeFIM, Wee Functional Independent Measures

Table 3. Effects of type of cerebral palsy on the GMFM, BSID-II, and WeeFIM ( $\mathrm{N}=68)$

\begin{tabular}{lcccc}
\hline Type & GMFM & $\begin{array}{c}\text { BSID-II } \\
\text { Motor scale }\end{array}$ & $\begin{array}{c}\text { BSID-II } \\
\text { Cognitive scale }\end{array}$ & WeeFIM $^{*}$ \\
\hline Spastic hemiplegia & $39.7 \pm 26.4$ & $43.6 \pm 19.6$ & $84.9 \pm 41.5$ & $99.4 \pm 17.2$ \\
Spastic diplegia & $49.6 \pm 27.9$ & $55.3 \pm 25.7$ & $93.1 \pm 41.0$ & $79.2 \pm 21.5$ \\
Spastic quadriplegia & $47.6 \pm 30.1$ & $57.4 \pm 26.8$ & $104.7 \pm 41.4$ & $49.6 \pm 12.8$ \\
Dystonia & $45.1 \pm 26.8$ & $51.9 \pm 26.0$ & $87.6 \pm 38.2$ & $45.8 \pm 20.1$ \\
\hline
\end{tabular}

Abbreviations are the same as in Table 2

$* \mathrm{p}<0.05$

Table 4. Effects of ability to stand with/without assistance on the GMFM, BSID-II, and WeeFIM (N=68)

\begin{tabular}{lcccc}
\hline Standing & GMFM $^{*}$ & $\begin{array}{c}\text { BSID-II } \\
\text { Motor scale }\end{array}$ & $\begin{array}{c}\text { BSID-II } \\
\text { Cognitive scale }\end{array}$ & WeeFIM \\
\hline Independent standing & $73.8 \pm 15.1$ & $74.9 \pm 18.0$ & $121.0 \pm 32.9$ & $71.9 \pm 26.8$ \\
Assistive standing & $25.8 \pm 14.2$ & $37.1 \pm 15.5$ & $75.6 \pm 35.1$ & $65.2 \pm 27.2$ \\
\hline
\end{tabular}

Abbreviations are the same as in Table 2

$* \mathrm{p}<0.05$

Table 5. Comparisons of GMFM, BSID-II, and WeeFIM ( $\mathrm{N}=68)$

\begin{tabular}{lcccc}
\hline & GMFM & $\begin{array}{c}\text { BSID-II } \\
\text { Motor scale }\end{array}$ & $\begin{array}{c}\text { BSID-II } \\
\text { Cognitive scale }\end{array}$ & WeeFIM \\
\hline GMFM & & $0.922^{* * *}$ & $0.719^{* * *}$ & 0.044 \\
BSID-II Motor scale & $0.922^{* * *}$ & & $0.866^{* * *}$ & -0.035 \\
BSID-II Cognitive scale & $0.719^{* * *}$ & $0.866^{* * *}$ & & -0.055 \\
WeeFIM & 0.044 & -0.035 & -0.055 & \\
\hline
\end{tabular}

Abbreviations are the same as in Table 2

$* * * \mathrm{p}<0.001$

and the ability to stand with or without assistance on physical and cognitive functioning, and ADL, and the relationship between physical and cognitive functioning in children with CP. The main findings of this study were: physical and cognitive functions significantly differed between standing with assistance and standing without assistance; ADL was significantly affected by CP type; and GMFM scores were significantly correlated with the BSID-II motor and cognitive scales, but not with WeeFIM.

Age appropriate capabilities for growth and development are certainly important for functional activities as well as independent daily and social living for children with CP. However, balanced development of physical and cognitive functioning is rare in children with $\mathrm{CP}$. If children with $\mathrm{CP}$ do not function normally in a specific domain, they do not show normal overall development, even though their development might be close to normal in other domains. Temporal growth and development might be close to normal in other domains. Temporal growth and development can be affected by abnormal posture, muscle and joint contractures, malformation, visual impairment, sensory impairment, and other major dysfunctions ${ }^{10}$. Most studies of children with neurological disorders have focused on their physical consequences, and have used cognitive and perceptual functions to evaluate the effectiveness and determine the appropriateness of therapeutic interventions in rehabilitation. It is important to examine cognitive impairment in $\mathrm{CP}$, because cognitive deficits independently affect the performance of 
functional activities and integration with community and school surroundings ${ }^{11)}$. However, there has been a paucity of study on the relationship between physical and cognitive functioning in children with CP and only a few studies have demonstrated significant relationships between physical and cognitive functioning.

This study analyzed the effects of age, CP type, and ability to stand independently or with assistance on motor function, cognitive function, and ADL of children with CP. The results indicate that ADL are significantly affected by type of CP, but not by age or standing independence. However, physical and cognitive functions were significantly affected by type of $\mathrm{CP}$ and independent standing. The results suggest that physical functioning is positively related with cognitive functioning in children with $\mathrm{CP}$, since children who moved more actively showed higher scores in terms of physical and cognitive functioning than the children who showed fewer active movements. Therefore, to plan therapeutic exercise for $\mathrm{CP}$, therapists should consider the effects of active movement, such as standing, on physical and cognitive functions and the relationship between physical and cognitive functioning.

In conclusion, the present study provides evidence of the necessity of including cognitive and physical impairments in the examination and evaluation of $\mathrm{CP}$ in research and clinical settings, because our results show that cognitive function is positively related with physical function in children with CP. This study did not show a positive correlation between ADL and physical and cognitive functioning. Future studies will need to investigate these correlations.

\section{REFERENCES}

1) Krigger KW: Cerebral palsy: an overview. Am Fam Physician, 2006, 73 91-100. [Medline]

2) Maruishi M, Mano Y, Sasaki T, et al.: Cerebal palsy in adults: independent effects of muscle strength and muscle tone. Arch Phys Med Rehabil, 2001, 82: 637-641. [Medline] [CrossRef]

3) Novacheck TF, Trost JP, Sohrweide S: Examination of the child with cerebral palsy. Orthop Clin North Am, 2010, 41: 469-488. [Medline] [CrossRef

4) Stanger M, Oresic S: Rehabilitation approaches for children with cerebral palsy: overview. J Child Neurol, 2003, 18: S79-S88. [Medline]

5) Romeo DM, Cioni M, Battaglia LR, et al.: Spectrum of gross motor and cognitive functions in children with cerebral palsy: gender difference. Eur J Paediatr Neurol, 2011, 15: 53-58. [Medline] [CrossRef]

6) Harvey A, Robin J, Morris M, et al.: A systematic review of measures of activity limitation for children with cerebral palsy. Dev Med Child Neurol, 2008, 50: 190-198. [Medline] [CrossRef]

7) Wei S, Wang SJ, Liao YG, et al.: Reliability and validity of the GMFM-66 in 0- to 3-year-old children with cerebral palsy. Am J Phys Med Rehabil, 2006, 85: 141-147. [Medline]

8) Connolly BH, Dalton L, Smith JB, et al.: Concurrent validity of the Bayley scales of infant development II (BSID-II) motor scale and the Peabody developmental motor scale II (PDMS-2) in 12-months-old infants. Pediatr Phys Ther, 2006, 18, 190-196.

9) Park EY, Kim WH, Choi YI: Factor analysis of the WeeFIM in children with spastic cerebral palsy. Disabil Rehabil, 2012 (in press).

10) Franki I, Desloovere K, De Cat J, et al.: The evidence-base for conceptual approaches and additional therapies targeting lower limb function in children with cerebral palsy: a systematic review using the ICF as a framework. J Rehabil Med, 2012, 44: 396-405. [Medline] [CrossRef]

11) Hadden KL, von Baeyer CL: Clobal and specific behavioral measures of pain in children with cerebral palsy. Clin J Pain, 2005, 21: 140-146. [Medline] [CrossRef] 